\title{
Effect of tourniquet application during blood sampling on RBC deformability and aggregation: Is it better to keep it on?
}

\author{
Philippe Connes ${ }^{\mathrm{a}, *}$, Norbert Nemeth ${ }^{\mathrm{b}}$, Herbert J. Meiselman ${ }^{\mathrm{c}}$ and Oguz K. Baskurt ${ }^{\mathrm{d}}$ \\ ${ }^{a}$ Laboratory ACTES (EA 3596), Department of Physiology, University of the French West Indies, \\ Guadeloupe \\ ${ }^{\mathrm{b}}$ Department of Operative Techniques and Surgical Research, Institute of Surgery, Medical and Health \\ Science Centre, University of Debrecen, Debrecen, Hungary \\ ${ }^{\mathrm{c}}$ Department of Physiology and Biophysics, Keck School of Medicine, Los Angeles, CA, USA \\ ${ }^{\mathrm{d}}$ Department of Physiology, Faculty of Medicine, Akdeniz University, Antalya, Turkey
}

\begin{abstract}
We have recently demonstrated that the use of a tourniquet to locate the vein prior to blood sampling may affect red blood cell (RBC) deformability and aggregation, even if the tourniquet is quickly removed after insertion of the needle into the vein. However, in clinical practice, the tourniquet is usually kept in place until blood sampling is completed. The present study tested the effects of tourniquet application during the blood sampling period on RBC deformability and aggregation. We compared blood samples obtained at 5, 30, 60 and 90 seconds following the tourniquet application; a control blood sample was obtained without applying a tourniquet. RBC deformability was slightly increased at 5 seconds $(7.6 \%$ mean, $1-5 \mathrm{~Pa})$ and at 60 seconds $(5.9 \%$ mean, $1-30 \mathrm{~Pa}$ ) but not at 30 or 90 seconds, with the increase inversely related to shear stress. The RBC aggregation index was slightly but significantly decreased at 5, 30 and 60 seconds, with no changes observed for either amplitude or half-time of aggregation. These very modest yet significant effects of tourniquet application on hemorheological parameters may not be meaningful in terms of pathophysiology, but may become important in data interpretation or for specific subjects.
\end{abstract}

Keywords: Red blood cell deformability, red blood cell aggregation, methodology, venipuncture procedures, blood sampling

\section{Introduction}

In 1986 an Expert Committee on Blood Rheology set international guidelines for measuring blood viscosity and red blood cell (RBC) deformability [3]. However, many technical aspects and devices have been developed since the publication of that report, and thus there is now a need to revise these previous guidelines. Therefore, a new expert panel has been formed for this purpose [1] and several experimental studies have been carried out to determine the best laboratory practice in hemorheology.

\footnotetext{
${ }^{*}$ Corresponding author: Philippe Connes, PhD, Laboratory ACTES (EA 3596), Department of Physiology, Campus de Fouillole, University of the French West, Guadeloupe. E-mail: pconnes@yahoo.fr.
} 
One aspect of the guidelines deals with blood sampling for hemorheological measurements. The previous guidelines stated that: "a tourniquet should be used only to allow localization of the vein and insertion of the needle and the tourniquet must be removed for at least five seconds prior to actual blood withdrawal" [3]. However, in a recent study, it has been observed that the effect of tourniquet application for the localization of vein and insertion of the needle may continue for several minutes after the removal of tourniquet [4]. In brief, both RBC deformability and aggregation were found to be affected in samples obtained during the $90 \mathrm{~s}$ period after the tourniquet removal, suggesting that the removal of the tourniquet just before sampling might cause spurious results. Obviously, an alternative to this practice is to keep the tourniquet in place until the blood sampling is completed [8]. The present study tested the effects of tourniquet application during the blood sampling period on RBC deformability and aggregation.

\section{Materials and methods}

\subsection{Study design}

The study was conducted with 10 healthy, male volunteers, all of whom gave written informed consent. A tourniquet was applied to the left arm to locate the antecubital veins and insert the needle. The time between the application of the tourniquet and the insertion of the needle into the vein was $15 \mathrm{~s}$. Blood samples were obtained into four $5 \mathrm{ml}$ vacuum tubes containing ethylendiaminetetraaceticacid (EDTA; $1.5 \mathrm{mg} / \mathrm{ml}$ ) at 5, 30, 60 and $90 \mathrm{~s}$ following insertion of the needle. The tourniquet was kept in place during the entire period of blood sampling. Another sample which served as a control was obtained from an antecubital vein on the other arm without applying a tourniquet. All subjects were sampled while quietly sitting and all venipunctures were done by one experienced technician.

\subsection{Hematological/hemorheological measurements}

All measurements were performed using the tubes in random order.

Mean corpuscular volume (MCV) and mean corpuscular hemoglobin concentration (MCHC) were measured using an electronic hematology analyzer (Max M-Retic, Coulter, USA). RBC deformability, as an Elongation Index (EI), was measured at $37^{\circ} \mathrm{C}$ and various fluid shear stresses by laser diffraction analysis using an ektacytometer (LORCA, RR Mechatronics, Hoorn, The Netherlands) [7]. EI values were determined at nine shear stresses ranging from 0.30 to $30 \mathrm{~Pa}$, with two methods utilized to report results: (1) tabular for each shear stress; (2) use of a Lineweaver-Burk approach for linerizing EI-stress data to determine the shear stress required for half-maximal deformation $\left(\mathrm{SS}_{1 / 2}\right)$ and the theoretical maximum EI at infinite shear stress $\left(\mathrm{EI}_{\max }\right)$ [2]. Note that increased $\mathrm{SS}_{1 / 2}$ values indicate decreased $\mathrm{RBC}$ deformability and, since all nine stress levels are used, provide an overall appreciation of experimental results. The reproducibility of the deformability method, as indexed by the coefficient of variation (CV) for 10 measures, ranges between $0.2 \%$ and $3.3 \%$ at high and low shear stress.

The LORCA was also used to determine various indices of RBC aggregation: (1) RBC aggregation index (AI; $\mathrm{CV}=2.5 \%)$; (2) minimal shear rate to prevent aggregation $\left(\gamma_{\mathrm{Tmin}}\right)$; (3) amplitude of signal change during aggregation (AMP; $\mathrm{CV}=4.2 \%)$; (4) half-time for aggregation $(\mathrm{CV}=4.1 \%)$ [5,7]. 


\subsection{Statistical analyses}

Values are presented as mean \pm standard deviation. For EI data, a two way ANOVA for repeated measures (shear stress $\times$ time) was used for statistical comparisons. A one way analysis of variance (ANOVA) for repeated measures was used for comparisons of the other parameters; the Tukey posthoc test was used as necessary. Statistical significance was established at $p<0.05$. All analyses were conducted using Statistica (v. 5.5, Statsoft, USA).

\section{Results}

\subsection{Hematology}

Hematocrit, MCV and MCHC did not significantly differ from control at any sampling time point (data not shown).

\section{2. $R B C$ deformability}

As shown in Table 1, EI was mainly affected at 5 and $60 \mathrm{~s}$ after tourniquet application, with no effect noted at $30 \mathrm{~s}$. All significant changes of EI were increased above control, and ranged from $12 \%$ at $0.95 \mathrm{~Pa}$ to $4 \%$ at $30 \mathrm{~Pa}$. At $90 \mathrm{~s}$ of tourniquet application, EI was altered only at the highest shear stress. Neither $\mathrm{SS}_{1 / 2}$ (Fig. 1(A)) nor EI $\mathrm{I}_{\max }$ (Fig. 1(B)) were significantly altered for any samples obtained during tourniquet application.

\subsection{RBC aggregation}

The aggregation index AI was slightly but significantly decreased for the samples obtained at 5,30 and $60 \mathrm{~s}$ after the tourniquet application (Fig. 2). No significant changes were observed for the amplitude of RBC aggregation (AMP), the half-time for RBC aggregation or the disaggregation shear rate $\left(\gamma_{\mathrm{Tmin}}\right)$ (data not shown).

Table 1

Comparisons of Elongation Index (EI) for control versus 5-90 s after venipuncture with tourniquet kept in place

\begin{tabular}{lccccc}
\hline SS (Pa) & Control & $5 \mathrm{~s}$ & $30 \mathrm{~s}$ & $60 \mathrm{~s}$ & $90 \mathrm{~s}$ \\
\hline 0.3 & $0.035 \pm 0.013$ & $0.039 \pm 0.014$ & $0.031 \pm 0.008$ & $0.038 \pm 0.017$ & $0.036 \pm 0.011$ \\
0.53 & $0.076 \pm 0.018$ & $0.090 \pm 0.022$ & $0.084 \pm 0.017$ & $0.092 \pm 0.022$ & $0.088 \pm 0.015$ \\
0.95 & $0.159 \pm 0.025$ & $0.178 \pm 0.026^{*}$ & $0.173 \pm 0.026$ & $0.178 \pm 0.027^{*}$ & $0.175 \pm 0.017$ \\
1.69 & $0.256 \pm 0.026$ & $0.277 \pm 0.027^{*}$ & $0.272 \pm 0.029$ & $0.277 \pm 0.027^{*}$ & $0.274 \pm 0.017$ \\
3 & $0.358 \pm 0.031$ & $0.381 \pm 0.026^{*}$ & $0.376 \pm 0.032$ & $0.378 \pm 0.027^{*}$ & $0.375 \pm 0.020$ \\
5.33 & $0.446 \pm 0.029$ & $0.466 \pm 0.023^{*}$ & $0.463 \pm 0.029$ & $0.465 \pm 0.024^{*}$ & $0.463 \pm 0.018$ \\
9.48 & $0.507 \pm 0.019$ & $0.549 \pm 0.020$ & $0.522 \pm 0.022$ & $0.527 \pm 0.018^{*}$ & $0.524 \pm 0.012$ \\
16.91 & $0.551 \pm 0.015$ & $0.566 \pm 0.022$ & $0.563 \pm 0.021$ & $0.571 \pm 0.015^{*}$ & $0.569 \pm 0.009$ \\
30 & $0.582 \pm 0.018$ & $0.596 \pm 0.027$ & $0.593 \pm 0.026$ & $0.605 \pm 0.013^{*}$ & $0.602 \pm 0.011^{*}$ \\
\hline
\end{tabular}

Notes: Values are means $\pm \mathrm{SD}, \mathrm{SS}=$ shear stress.

${ }^{*}$ Difference from control $(p<0.05)$. 


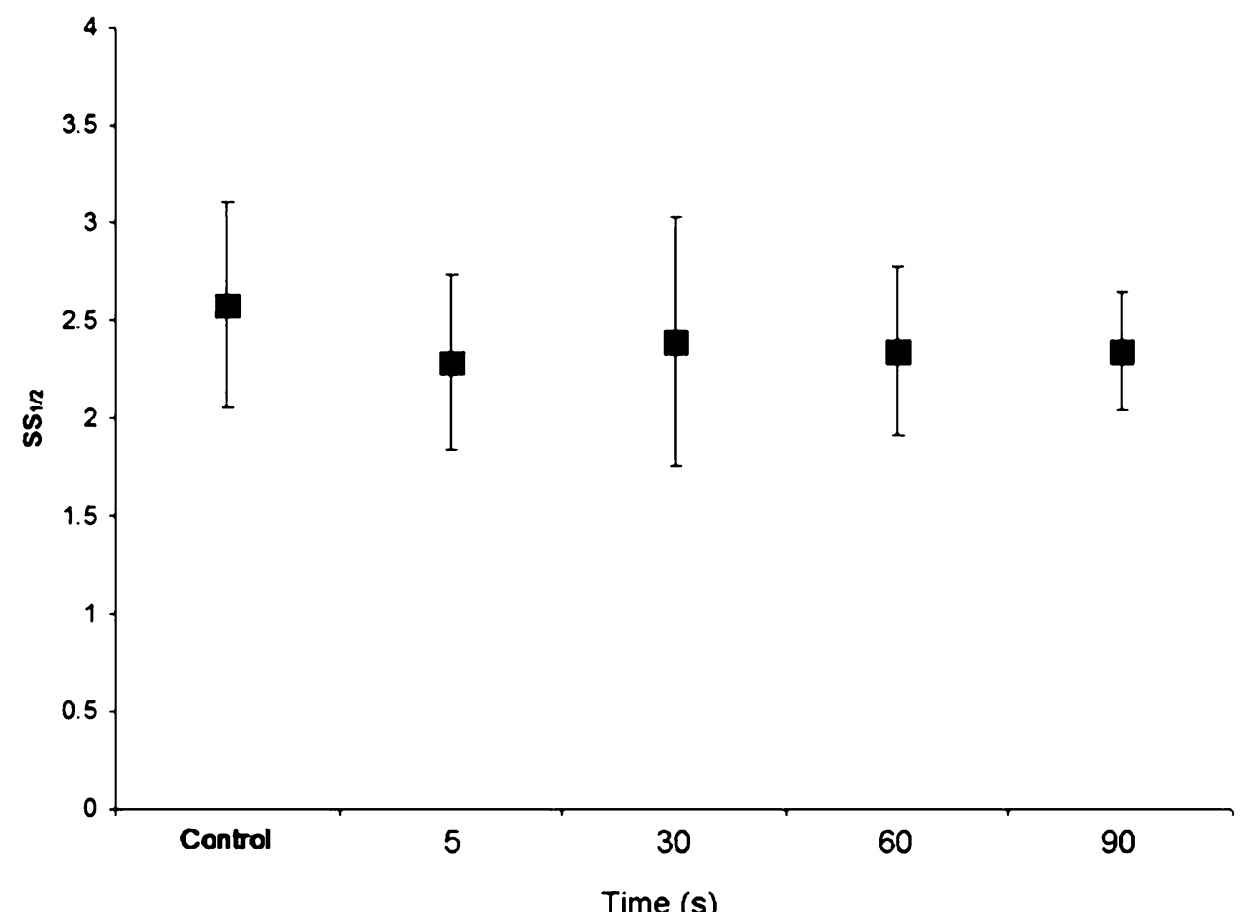

(A)

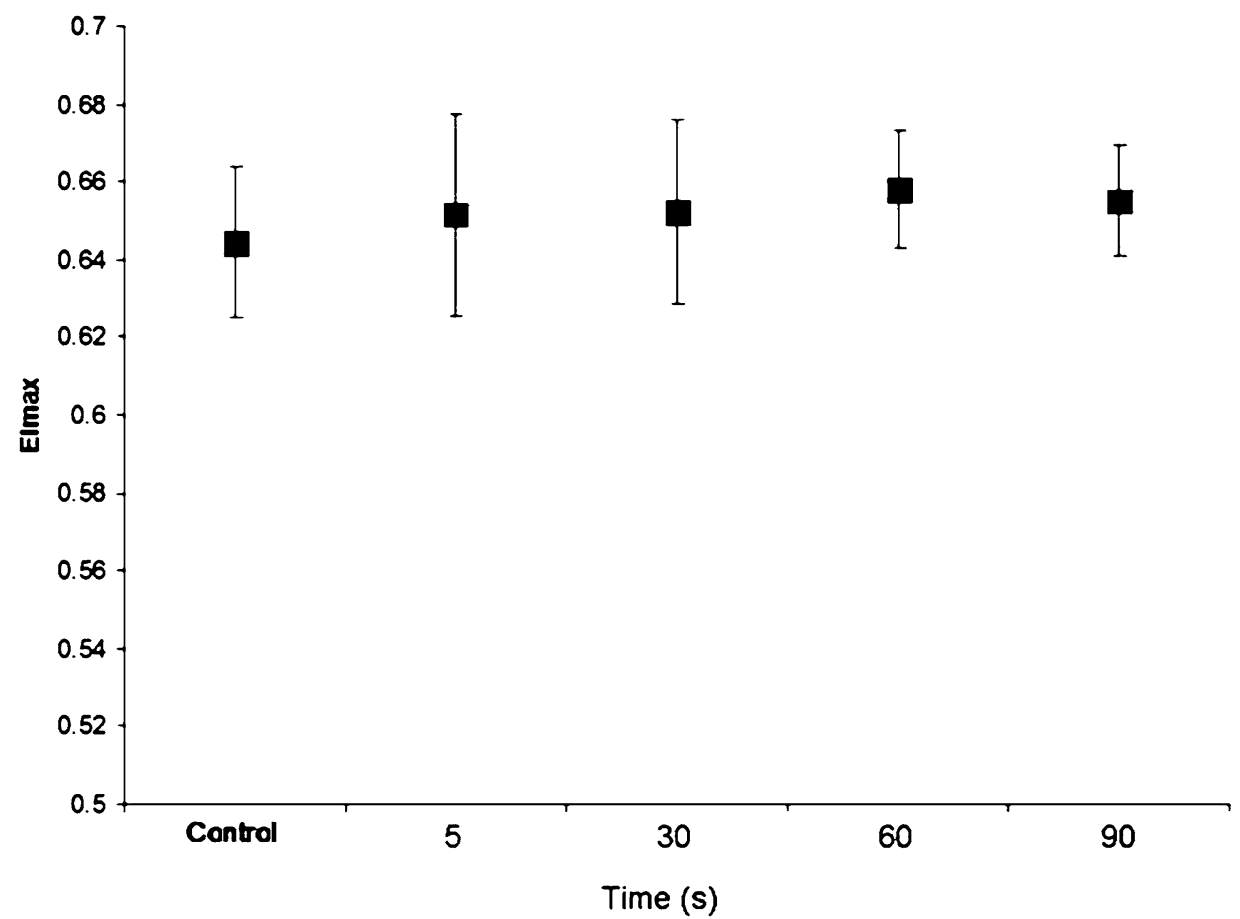

(B)

Fig. 1. (A) Shear stress required for half-maximal $\mathrm{RBC}$ deformation $\left(\mathrm{SS}_{1 / 2}\right)$, (B) theoretical maximum EI at infinite shear stress $\left(E I_{\max }\right)$ for control and for samples obtained at times after venipuncture with tourniquet applied. 


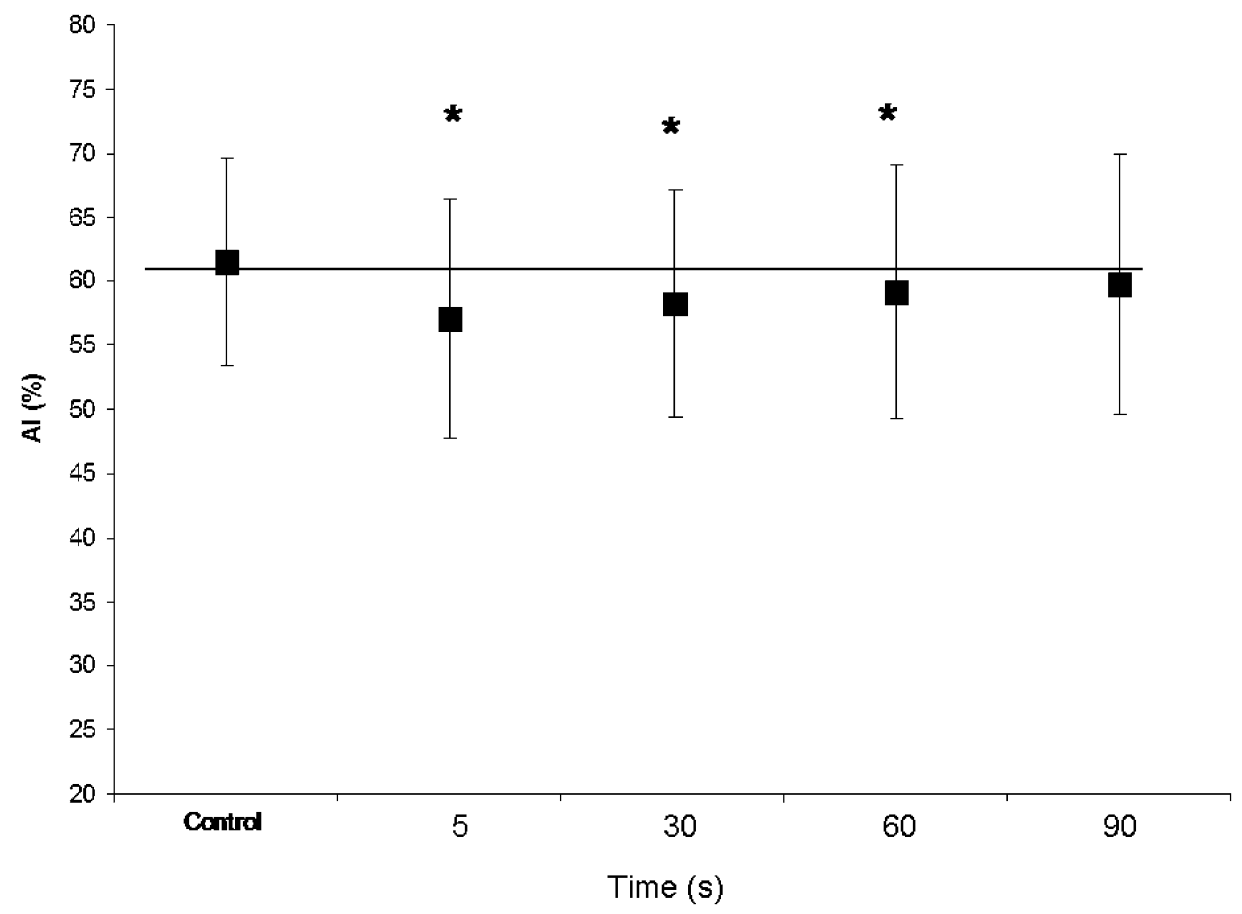

Fig. 2. Aggregation index (AI) for control and for samples obtained at times after venipuncture with tourniquet applied. * Significant difference from control, $p<0.05$.

\section{Discussion}

Our results indicate that both $\mathrm{RBC}$ aggregation and deformability can be affected by the application of tourniquet during blood sampling. Significant increases of RBC deformability were observed at three time points and at several levels of shear stress, with more marked changes at $60 \mathrm{~s}$ (Table 1). However, four aspects of these results merit attention: (1) the increases were, in general, small (4-12\%) with the greatest differences noted at the lower stress levels; (2) no changes of deformability were noted for samples drawn at $30 \mathrm{~s}$; (3) neither $\mathrm{SS}_{1 / 2}$ nor EImax were significantly affected by the duration between tourniquet application and blood sampling; (4) the changes observed herein are quite similar to those observed when the tourniquet was removed just prior to sampling [4]. In addition, the significant changes of RBC aggregation were also modest (5-8\%) as in the previous study [4], with the greatest difference observed at $5 \mathrm{~s}$.

The practice of removing the tourniquet before the sampling is not a universal procedure in clinical medicine. Rather, it is very common to leave the tourniquet in place until the phlebotomy is completed [8], even though this approach can induce changes in the sampled blood. Lippi et al. [9,10] demonstrated that tourniquet time ( $1 \mathrm{~min}$ versus $3 \mathrm{~min}$ ) may strongly affect the values of routine hematological and coagulation parameters. In addition, it has been demonstrated that prolonged application ( $>3 \mathrm{~min}$ ) of a tourniquet during sampling may affect plasma viscosity [11] and blood viscosity [6].

In conclusion, there is evidence for very modest yet significant effects of tourniquet application on hemorheological parameters. These effects occur regardless of whether the tourniquet is removed prior to the blood sampling or it is left in place, and are observed at different time points for each tourniquet procedure. While the alterations may not be meaningful in terms of pathophysiology, they are statisti- 
cally significant changes that may become important in data interpretation. Further, it should be noted that the effect of tourniquet application may be different in specific groups of patients (e.g., sickle cell disease) in which the response of RBC to brief hypoxia might be different from normal subjects.

In summary, it is strongly recommended that the selected blood sampling procedure (with/without tourniquet application, with/without removal prior to sampling, timing of samples) should be carefully described and that this procedure should be utilized for all subjects.

\section{References}

[1] O.K. Baskurt, M. Boynard, G. Cokelet, P. Connes, B.M. Cooke, M.R. Hardeman, F. Liao, H.J. Meiselman, G.B. Nash, N. Nemeth, B. Sandhagen, S. Shin, G.B. Thurston, J.L. Wautier and S. Yedgar, New guidelines for the assesment of hemorheological parameters, Biorheology 45 (2008), 82.

[2] O.K. Baskurt and H.J. Meiselman, Analyzing shear stress-elongation index curves: Comparison of two approaches to simplify data presentation, Clin. Hemorheol. Microcirc. 31 (2004), 23-30.

[3] B.S. Bull, S. Chien, J.A. Dormandy, H. Kiesewetter, S.M. Lewis, G.D.O. Lowe, H.J. Meiselman, S.B. Shohet, J.F. Stoltz, J. Stuart and P. Teitel, Guidelines for measurement of blood viscosity and erythrocyte deformability, International Committee for Standardization in Haematology. Expert panel on blood rheology, Clin. Hemorheol. 6 (1986), 439-453.

[4] P. Connes, M. Uyuklu, J. Tripette, J.H. Boucher, E. Beltan, T. Chalabi, O. Yalcin, R. Chout, O. Hue, M.D. HardyDessources and O.K. Baskurt, Sampling time after tourniquet removal affects erythrocyte deformability and aggregation measurements, Clin. Hemorheol. Microcirc. 41(1) (2009), 9-15.

[5] J.G.G. Dobbe, G.J. Streekstra, J. Strackee, M.C.M. Rutten, J.M.A. Stijnen and C.A. Grimbergen, Syllectometry: The effect of aggregometer geometry in the assessment of red blood cell shape recovery and aggregation, IEEE Trans. Biomed. Eng. 50 (2003), 97-106.

[6] S. Forconi, Hemorheological changes during venous stasis as result of tourniquet application, Clin. Hemorheol. Microcirc. 20 (1999), 197-199.

[7] M.R. Hardeman, J.G.G. Dobbe and C. Ince, The Laser-assisted Optical Rotational Cell Analyzer (LORCA) as red blood cell aggregometer, Clin. Hemorheol. Microcirc. 25 (2001), 1-11.

[8] I. Lavery and P. Ingram, Venepuncture: best practice, Nurs. Stand. 19 (2005), 55-65; quiz 66.

[9] G. Lippi, G.L. Salvagno, M. Montagnana and G.C. Guidi, Short-term venous stasis influences routine coagulation testing, Blood Coagul. Fibrinol. 16 (2005), 453-458.

[10] G. Lippi, G.L. Salvagno, G.P. Solero and G.C. Guidi, The influence of the tourniquet time on hematological testing for antidoping purposes, Int. J. Sports Med. 27 (2006), 359-362.

[11] R.S. Rosenson and C.C. Tangney, Effects of tourniquet application on plasma viscosity measurements, Clin. Hemorheol. Microcirc. 18 (1998), 191-194. 